\title{
The Implementation of Real-time Rendering for Elbow Training in AR-based Rehabilitation System
}

\author{
Qinghao Cao ${ }^{1, a}$, Qiang Wang ${ }^{1, b}$, Bingjin $\mathrm{Ji}^{2, c^{*}}$ and Xiao $\mathrm{Li}^{1, \mathrm{~d}^{*}}$ \\ ${ }^{1}$ School of Computer and Information Science, Southwest University, Chongqing, 400700, China \\ ${ }^{2}$ Beibei Traditional Chinese Medical Hospital, Chongqing, 400700, China \\ a497326856@qq.com, ${ }^{\text {b wqwq1219@163.com, }{ }^{\text {c5}} 570676078 @ q q . c o m, ~}$ \\ dixiaoatswu@139.com
}

Keywords: AR; Real-time Rendering; Rehabilitation System; elbow

\begin{abstract}
Elbow movement disorder is one of the common diseases affecting the daily activities of patients. Augmented Reality (AR) technology fuses the virtual information generated by computer with the real environment, keeping the patients' real-world feeling and also helping to training evaluation. This paper mainly deals with another key technology; real-time rendering of elbow virtual model in three-dimensional space, on the basis of the team has solved the key problem of registration tracking of AR-based upper limb rehabilitation evaluation training. By contrasting and analyzing the existing four rendering technologies, a new solution is put forward. The ideas are as follows: the design of the scene rendering module, the design of the related algorithms. System prototype expected effect real-time rendering during elbow evaluation training.
\end{abstract}

\section{Introduction}

Elbow joint is a part of the upper limb, which plays an important role for daily activities [1]. Elbow movement disorder becomes more common with the frequency of various diseases or accidents; it needs to be treated with training rehabilitation system [2]. Nowadays, one of training methods for rehabilitation is the use of virtual computer systems like Yikang Medical Equipment that can't monitor and evaluate the recovery process [3].

AR technology, which fuses the real environment with the virtual information generated by computer, is to enhance the real world [4]. AR technology is more secure and easier to implement than VR technology. The system that utilize AR technology constructs a virtual-reality fusion training environment to make patients keep in touch with the real world, which is helpful to improve the rehabilitation better. At present the team has solved the key problems of system architecture: registration tracking and the evaluation of rehabilitation effects, which based on AR and sensor technology. The system is designed to be able to evaluate the training in real time and then immediately reflect this evaluation in the training, which consists of real training scene, virtual training scene, the human-computer interaction and the generation and display of virtual-reality fusion scene [5].

The team has completed three parts of the designs which are real training scene, the generation and display of virtual-reality fusion scene and the human-computer interaction. This paper presents a real-time rendering solution of AR-based rehabilitation system for virtual training scene module. Android devices integrate a variety of sensors and it's easy to wear, so the system prototype is based on the Android platform. The rest of this paper is organized as follows. Section II introduces related work and particularly analyzes four real-time rendering technologies and points out the advantages and feasibility of surface-rendering. Section III puts forward the realization of rendering engine of 3D scene and suggests the real-time rendering algorithm and implementation process.

\section{Related Work}

The essence of real-time rendering is real-time calculation and output of graphic data. By using accelerated rendering and Circulation of the feedback, users can't see a single image and 
completely immerse in the experience of image process of dynamic continuous display [6].The core of real-time rendering technology for mobile devices is processing the problem of complex geometry data on resource-constrained mobile devices with high quality rendering [7]. Therefore, Table 1 respectively discusses four methods of real-time rendering include technical characteristics, interaction latency, display quality and data compression. Among them, interaction delay is to explore how to ensure the interaction delay within the allowable range of users; smooth interaction is the purpose of all the applications. Display quality means how to ensure that the mobile devices can provide acceptable rendering quality to users on a limited screen resolution. And data compression refers to how to simplify data, reduce the amount of data that is represented, stored, and transferred [8] [9] [10].

Table 1 The comparisons of four kinds of real-time rendering

\begin{tabular}{|c|c|c|c|c|}
\hline Categories & Technical Characteristics & $\begin{array}{c}\text { interaction } \\
\text { latency }\end{array}$ & display quality & $\begin{array}{c}\text { data } \\
\text { compression }\end{array}$ \\
\hline $\begin{array}{l}\text { Surface } \\
\text { rendering }\end{array}$ & $\begin{array}{l}\text { The method is based on the } \\
\text { description of contours, that } \\
\text { is, the object contours are } \\
\text { separated by manual or } \\
\text { automatic method and then } \\
\text { piled together the contours } \\
\text { of each layer in the } \\
\text { sectional image }\end{array}$ & $\begin{array}{l}\text { It has the } \\
\text { features of low } \\
\text { computational } \\
\text { cost, low } \\
\text { interaction } \\
\text { latency and } \\
\text { high running } \\
\text { speed }\end{array}$ & $\begin{array}{l}\text { The meaningful } \\
\text { visual information } \\
\text { is extracted from } \\
3 \mathrm{D} \text { data sets, and } \\
\text { the display quality } \\
\text { is higher }\end{array}$ & $\begin{array}{l}\text { The } \\
\text { representation } \\
\text { method is } \\
\text { simple, the size } \\
\text { of data is small } \\
\text { and the speed } \\
\text { of data } \\
\text { transmission is } \\
\text { fast }\end{array}$ \\
\hline $\begin{array}{l}\text { Volume } \\
\text { rendering. }\end{array}$ & $\begin{array}{l}\text { Discrete data of 3D data } \\
\text { sets which is used } \\
\text { two-dimensional image } \\
\text { draws and displays on the } \\
\text { screen by means of } \\
\text { three-dimensional voxel } \\
\text { without segmentation }\end{array}$ & $\begin{array}{l}\text { It needs to } \\
\text { process all the } \\
\text { voxels, the } \\
\text { image } \\
\text { rendering speed } \\
\text { is slower and } \\
\text { the delay is } \\
\text { higher }\end{array}$ & $\begin{array}{l}\text { The details of the } \\
3 \mathrm{D} \quad \text { image } \\
\text { information are } \\
\text { reserved which } \\
\text { have } \\
\text { high-quality } \\
\text { rendering a } \\
\text { display }\end{array}$ & $\begin{array}{l}\text { The } \\
\text { computation is } \\
\text { large with the } \\
\text { management of } \\
\text { volume data } \\
\text { shades and } \\
\text { the data } \\
\text { compression is } \\
\text { lower }\end{array}$ \\
\hline Model & $\begin{array}{l}\text { The object is represented by } \\
\text { an approximate shape } \\
\text { constructed by triangle } \\
\text { faces, and the model is } \\
\text { produced by 3DMax, Maya } \\
\text { and so on }\end{array}$ & $\begin{array}{l}\text { The rendering } \\
\text { which has a } \\
\text { high delay is } \\
\text { completed in } \\
\text { the process of } \\
\text { user interaction }\end{array}$ & $\begin{array}{l}\text { The edge of graph } \\
\text { is not smooth and } \\
\text { the display quality } \\
\text { is common }\end{array}$ & $\begin{array}{l}\text { It needs to } \\
\text { transmit a lot } \\
\text { of geometric } \\
\text { data and it's } \\
\text { difficult to } \\
\text { realize data } \\
\text { compression }\end{array}$ \\
\hline Particles & $\begin{array}{l}\text { It's namely point sprites } \\
\text { rendering which is } \\
\text { completed real-time } \\
\text { rendering by superimposing } \\
\text { constantly particles }\end{array}$ & $\begin{array}{l}\text { The efficiency } \\
\text { depends mainly } \\
\text { on the size of } \\
\text { the texture, and } \\
\text { the interaction } \\
\text { delay is } \\
\text { unstable }\end{array}$ & $\begin{array}{l}\text { It can't be drawn } \\
\text { according to the } \\
\text { angle of different } \\
\text { lights, and it has } \\
\text { the problem of } \\
\text { sorting alpha with } \\
\text { low display }\end{array}$ & $\begin{array}{l}\text { It's weaker } \\
\text { than model in } \\
\text { the } \\
\text { performance } \\
\text { and the data } \\
\text { compression is } \\
\text { also lower }\end{array}$ \\
\hline
\end{tabular}

Volume rendering is a vector method, which can describe the details and will not affect the efficiency. But the calculation is complex and the method needs some hardware supports, the efficiency in the Android device isn't high. Model rendering method takes the visual effect as the first modeling goal, the image tracking rendering is not so good for real-time, so the method can't complete the registration tracking of the elbow movement. The efficiency of particles rendering depends on the size of the sprite, the real-time direction of surface can't change which can't reflect the different angles of an object, so it's not apply to real-time rendering implementation of elbow 
training. The calculation of surface rendering method is small and the delay of interaction is low. The most important is that this method can solve the real-time positioning problems of the three-dimensional data sets with a good display quality.

AR-based rehabilitation system is a simulation of reality; the visual authenticity directly affects the feeling of patients and training effect, so it must adopt realistic algorithms of computer graphics such as shading technique, texture mapping, special effects and environment mapping. In particular, it can vividly record the unknown effect of elbow changes. When the traditional 3D modeling software is used for image modeling, the visual effect is usually used as the first purpose of the modeling [11].

In summary, Surface rendering is the best solution to solve real-time rendering of elbow training in AR rehabilitation system.

\section{Design Idea}

In order to fuse the virtual objects generated by the mobile platform with the surrounding environment really, it is necessary to render the embedded virtual objects with the photorealistic consistent environment. Sensors are detection devices that can monitor the data and transform it into other required forms in order to achieve the transmission, processing, storage and display [12]. This paper uses the method of real-time scene detection based on direction sensor, establishing the elbow model and virtual scene directly, constructs the virtual model in the operation of the system by three-dimensional dynamic data collected by sensor, and makes it complete the interaction of patients with elbow position changes.

The system prototype is mainly composed of 3D graphics engine and the training module under the engine, which is based on the Android platform and the common commercial graphics software. The principle structure of the system prototype is shown in Figure 1.

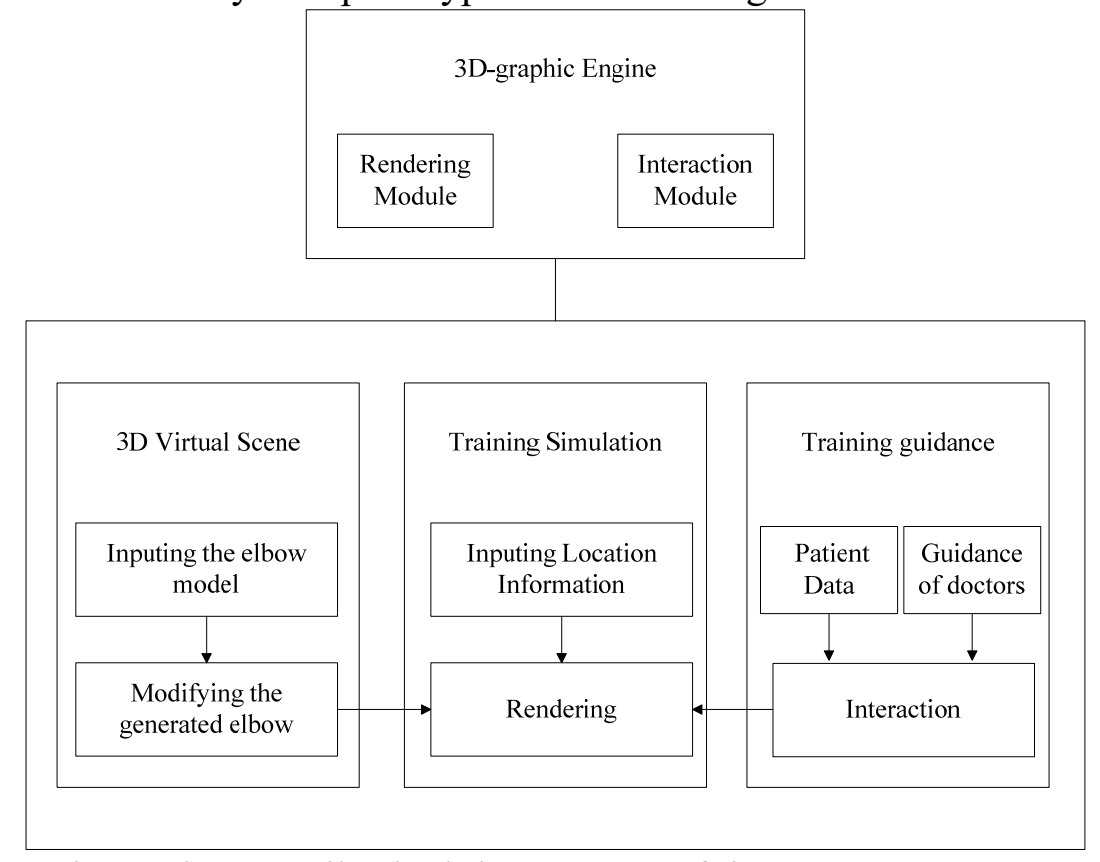

Fig.1. The overall principle structure of the system prototype

The system prototype with the aid of computer graphics and AR technology photorealistic renders the scenarios of elbow training on Android devices. Combining with the actual human frame, it builds up the real training scenarios which can interact AR training of patients. 3D-Graphic engine which models the elbow is the core of the whole system prototype based on object-oriented design. The relationship diagram of each function module and the rendering engine of the system prototype are shown in Figure 2. 


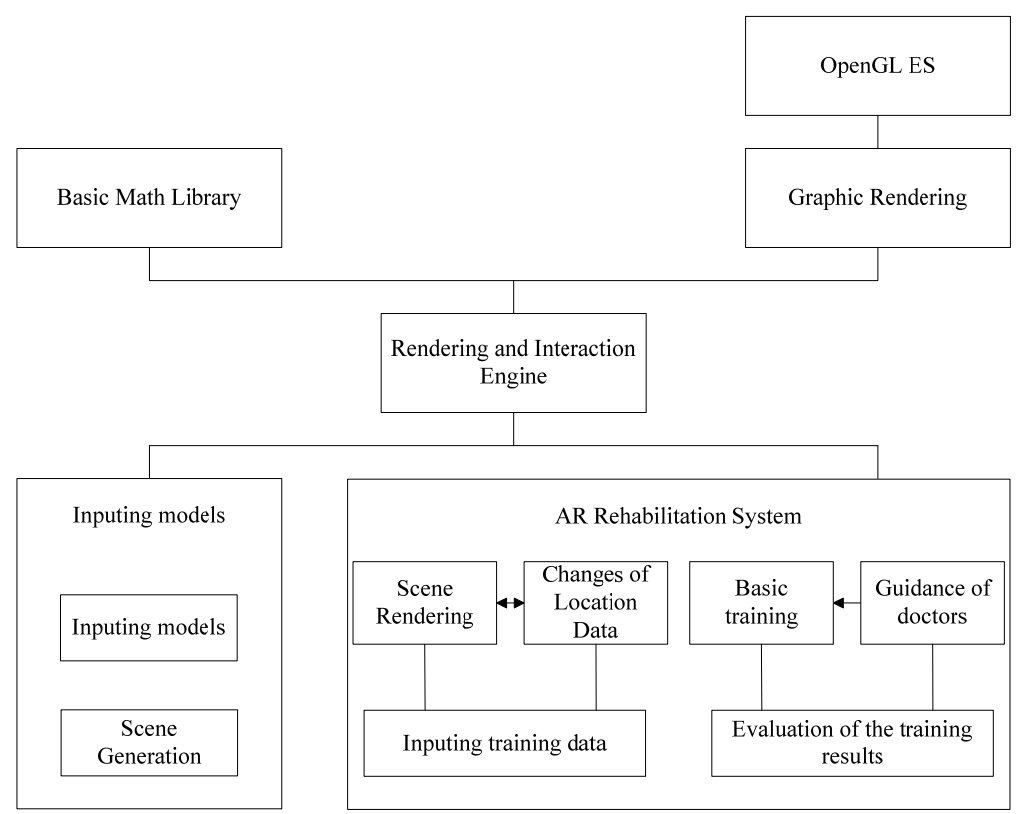

Fig.2. The module and function definition of the system prototype

The method which uses plug-in technology combines the three-dimensional rendering engine with the function module of the system prototype in the form of dynamic linking, which reduces the debugging complexity, facilitates the code reuse and facilitates the modular management. The function of system prototype is defined as follows.

3D-graphic engine. This is the core of the whole software to complete basic computer graphics algorithms, including basic mathematical operation, scene management and drawing, realistic algorithms and implementation, interaction and management of input devices.

Input module. The vertex data information of 3D model is output by OpenGL ES (OpenGL for Embedded Systems), and read by engine with the required file format to display.

Graphic interaction. It's used for scene rendering and scene interaction of patients, and it's composed of graphics rendering engine and graphics input module.

\section{Implementation Process}

Three processes which are APP, CULL and DRAW real-time scene rendering are used to calculate the location and direction of the virtual scene and model in the Android system. Using this method to build three-dimensional real-time rendering engine process of elbow model is showed in Figure 3.

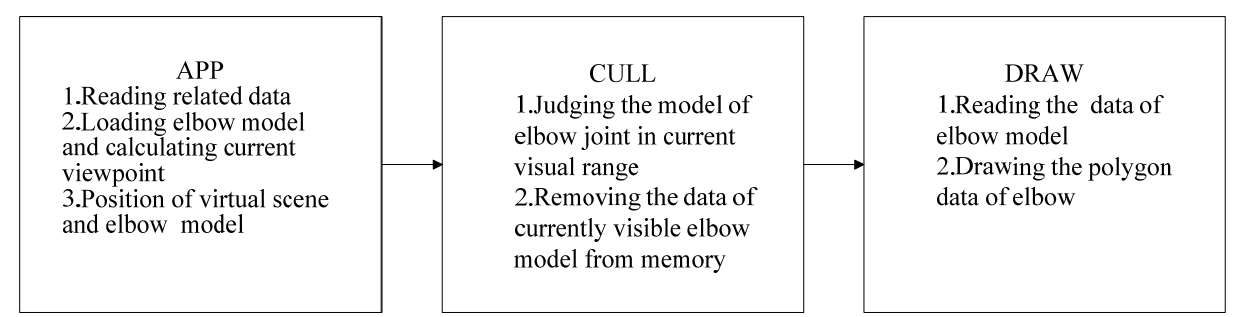

Fig.3. The process of rendering engine

The actual data of elbow movement parameters is collected according to the process of Figure 3 with special effect in the early.

Surface rendering is a technique of surface extraction and display. The 3D model of elbow is built by construction of the rendering engine according to Marching Cubes algorithm. The process of model building is as follows.

1) The $3 \mathrm{D}$ regular data field of the elbow model is constructed by the method of matching interpolation.

2) The surface of the elbow image is extracted. 
3) The appropriate threshold is determined, and the isosurface is extracted from the $3 \mathrm{D}$ data field according to the different gray scales of the elbow.

4) The model of elbow surface is displayed by the chiaroscuro technique of geometric surface in graphics.

Marching Cubes algorithm [13] is used to extract the isosurface of 3D data field. The basic unit is the cubic volume which is a logical cube, consisting of eight vertices on the cube which is composed of four points on the adjacent layer in marching cubes. The basic idea to extract the elbow isosurface of 3D data field is as follows: The cubes of the elbow model are processed one by one to estimate whether the eight vertices of the cube cross the isosurface in the data field. The interpolation is used to calculate the intersection of the isosurface and the cube edge for the cube crossing the isosurface. The intersections of the isosurface and the cube edge are connected in a certain way to generate the isosurface, as an approximation in the cube according to the relative position of each vertex of the cube and the isosurface.

The surface shading of the elbow model is calculated by Phong which is a surface shading model as shown below [14].

$$
\mathrm{I}=K_{\alpha} I_{\alpha}+K_{d} I_{l} \cos \theta+K_{s} I_{l} \cos ^{\mathrm{n}} \alpha
$$

Among them, $K_{\alpha}$ is constant which is related to the surface properties of the model; $I_{\alpha}$ is the luminous intensity of the incoming light; $\mathrm{K}_{\mathrm{d}}$ is diffuse constant which is related to the surface properties of the model; $I_{l}$ is the luminous intensity of the light source; $\theta$ is the angle between the incoming light and the normal vector of the surface; $\alpha$ is the angle between the line of sight and the normal vector of the elbow model; and $n$ is the symbol of the smoothness of the model surface.

Finally, a three-dimensional model of the elbow joint is built on the display device, which is a four-dimensional model (increasing the time dimension), namely the three-dimensional elbow model can be displayed in real-time.

\section{Validity Evaluation}

Users can be divided into three categories, namely healthy persons, simulate patients and patients to assess the effectiveness in order to test the security and usability of the system. Immersion of the system prototype determines participation enthusiasm of patients, how to keep the patients' attention in the AR environment is the standard of system immersion. So the system prototype should take into account the effect and efficiency of real-time rendering, and balance the rendering speed and effect.

The system prototype is mainly used to simulate the movement of patients during elbow training evaluation detect the ROM (range of motion) of elbow in real time. The model is simulated by the prototype which must be sensitive, precise, continuous and stable. The clinical availability and effectiveness of the systematic prototype is to be carried out by three test methods which are divided into healthy persons and simulate patients and patients in conjunction with the daily training of rehabilitation department. Consultation with the treatment of 8-10 objects will be selected to guide the evaluation training of the elbow rehabilitation, and the training results will be recorded in Table 2.

Table 2 The record of ROM

\begin{tabular}{|c|c|c|c|c|c|c|c|c|c|}
\hline Name: & Sex: & Number: & Diagn & is: & Hospita & Stays: & Disc & rge $\mathrm{Dc}$ & \\
\hline Joint & Inspection & $\begin{array}{c}\text { Reference } \\
\text { Value }\end{array}$ & & Date & Date & Date & Date & Date & Date \\
\hline \multirow{4}{*}{ Elbow } & \multirow[t]{2}{*}{ Flexion } & \multirow[t]{2}{*}{$0^{\circ} \sim 150^{\circ}$} & Left & & & & & & \\
\hline & & & Right & & & & & & \\
\hline & \multirow[t]{2}{*}{ Extension } & \multirow[t]{2}{*}{$0^{\circ} \sim 10^{\circ}$} & Left & & & & & & \\
\hline & & & Right & & & & & & \\
\hline
\end{tabular}

Patients are evaluated by the system prototype each time, and the next specific training goal is 
determined based on the evaluation results and the physician's opinion. The training is re-evaluated after the training; evaluation training of rehabilitation is a cyclical process.

\section{References}

[1] Shurong Ji. Rehabilitation Medicine [M]. Higher Education Press, 2006.

[2] Xiang Kong. The Research of System Based on Mobile Augmented Reality [D]. Xidian University, 2012.

[3] Kun Wang. The Research of Training Rehabilitation System Based on Augmented Reality [D]. Southeast University, 2013.

[4] Jia Liu and so on. The Research Development of Augmented Reality Technology in Rehabilitation of Manual Function [J]. Chinese Journal of Rehabilitation Medicine, 2014.

[5] Qiang Wang, Xiao Li, Lu Zeng and Qinghao Cao. Developing an AR-Based Upper Limb Rehabilitation System with Sensors [C]. The 2nd International Forum on Computers, Electronics and Mechatronics, 2015.491-497.

[6] Gilda Aparecida de Assis, Roseli de Deus Lopes, et al. Perception of Health Professional about Clinical Utility of an Augmented Reality Musical System to Motor and Cognitive Rehabilitation[C]. XVI Symposium on Virtual and Augmented Reality, 2014.71-79.

[7] Eisert P, Fechteler P. Low delay streaming of computer graphics[C]. Proceedings of IEEE International on Image Processing. Los Alarnitos, IEEE Computer Society Press, 2008.2704-2707.

[8] Chanchan Xu. A Survey of Remote Rendering Technology for Mobile Devices [J]. Journal of Computer-aided Design and Computer Graphics, 2016 (6) 914-922.

[9] Noguera J M, Segura R J, Ogayar C J. A scalable architecture for 3D map navigation on mobile devices [J]. Personal and Ubiquitous Computing, 201317 (7) 1487-1502.

[10] Yuping Wang. Reconstructing the Light Field of Human Face Based on Multi - view Images[J]. Journal of Beijing Polytechnic University, 2016 (6) 871-876.

[11] Xuepei Chen. Design and Realization of 3D Graphics Special Effect Algorithm in Game [D]. HUST, 2015.

[12] Anning Chen. Medical sensors, Second ed [M]. Science Publishing Ltd, 2008.

[13] Cline H, Lorensen W. Two Algorithms for the 3D Reconstruction of Tomsgrams [J]. Medical Physics, 199815 (3) 225-233.

[14] Guojun Ma. Voxel Classification and Phong Light Model GPU Accelerated Volume Rendering [J]. Computer Engineering and Design, 201031 (7) 1529-1532. 